\title{
WWC3 downregulation correlates with poor prognosis and inhibition of Hippo signaling in human gastric cancer
}

This article was published in the following Dove Press journal:

OncoTargets and Therapy

12 June 2017

Number of times this article has been viewed

\section{Jiabin Hou \\ Jin Zhou}

The First Affiliated Hospital, Harbin Medical University, Harbin, People's Republic of China
Correspondence: Jin Zhou

The First Affiliated Hospital, Harbin Medical University, 23 Youzheng Street, Harbin 15000 I, People's Republic of China

Email zhoujin I III@I26.com

\begin{abstract}
The aim of this study was to investigate the clinicopathological significance and biological roles of WWC3 in human gastric cancer (GC). Clinical significance of WWC3 in human GCs was examined by using immunohistochemistry (IHC). WWC3 was downregulated in 48 of 111 human GCs, and its downregulation was associated with advanced stage, positive nodal status, and higher relapse rate. Importantly, WWC3 downregulation correlated with poor survival. It was also found that WWC3 protein expression was downregulated in GC cell lines compared with normal cell line GES-1. On one hand, WWC3 overexpression inhibited the cell growth rate and invading ability in HGC-27 cell line. On the other hand, depleting WWC3 by small interfering RNA (siRNA) promoted proliferation rate and invading ability in the SGC-7901 cell line. In addition, cell cycle analysis showed that WWC3 overexpression inhibited while its depletion accelerated cell cycle progression at the G1/S transition. Western blot (WB) analysis demonstrated that WWC3 repressed cyclin D1 and cyclin E while upregulated p27 expression. Luciferase reporter assay showed that WWC3 activated Hippo signaling pathway by suppressing TEAD transcription activity, with downregulation of total and nuclear YAP and its target CTGF. WWC3 siRNA depletion exhibited the opposite effects. In conclusion, this study indicates that WWC3 serves as a tumor suppressor in GC by activating Hippo signaling.
\end{abstract}

Keywords: WWC3, gastric cancer, cell cycle, Hippo, YAP

\section{Introduction}

Gastric cancer (GC) is one of the most common malignant tumors of the digestive system and the third most common cause of cancer-related death in the People's Republic of China. ${ }^{1}$ Various complex genetic and epigenetic changes play key roles in its carcinogenesis and progression. Therefore, it is of great importance to identify effective molecular targets influencing tumor invasion and proliferation, which could be used as markers to predict the risk of $\mathrm{GC}^{2-7}$

WWC3, also known as WW and C2 domain containing protein 3, belongs to WWC scaffold protein family involved in cellular transport processes crucial for cell migration, polarity, and synaptic signaling. ${ }^{8-10}$ High endogenous expression of WWC3 has been shown in human ovarian and lung tissues. ${ }^{11}$ The role of WWC family proteins in human cancer development is gaining attention in recent years. Reduced WWC1/KIBRA expression was found in primary breast cancer specimens, which correlates with malignant subtype. ${ }^{12}$ WWC1 functions as a tumor suppressor by activating LATS kinase, which in turn prevents YAP nuclear import and cancer cell growth. ${ }^{12-14}$ WWC1 methylation occurs frequently in B-cell acute lymphocytic leukemia but not in epithelial cancers. ${ }^{15}$ It was reported that WWC1 methylation was associated with unfavorable biological 
prognostic parameters in chronic lymphocytic leukemia. ${ }^{16}$ WWC2 is able to form homo- and heterodimeric complexes with WWC1/3. Overexpression of WWC2 in HEK293T cells activated LATS1 phosphorylation. ${ }^{11}$

Although the function of KIBRA/WWC1 was studied intensively in animal models and human cancers, the importance of WWC3 was not yet elucidated. Since WWC1 and WWC3 share a similar structure, it is possible that WWC3 also functions as a tumor suppressor. To address the above question, this study explored protein expression of WWC3 in 111 cases of GC specimens and analyzed its clinical significance. Then, the association of WWC3 with malignant biological behavior of gastric cell lines was examined. In addition, the possible mechanism by which WWC3 regulated cell proliferation and invasion was investigated.

\section{Patients and methods}

\section{Patients and specimen}

This study protocol was approved by the institutional review board of The First Affiliated Hospital of Harbin Medical University. A total of 111 cases of gastric tumor specimens and 15 adjacent normal gastric tissues were obtained from patients diagnosed with GC who underwent resection in The First Affiliated Hospital of Harbin Medical University between 2007 and 2013. Written informed consent was obtained from the patients whose tissue samples and medical records were used and reviewed in this research.

Clinical and histopathological data including histopathological diagnosis and tumor grade were extracted from the medical records of the patients. All cases in this study received radical total or subtotal gastrectomy with D2/D3 lymph node dissection. Pathological examination revealed no tumor involvement of the resection margins in surgical specimens (R0: no residual tumor based on R category in AJCC classification). None of the patients included in this study had received preoperative chemotherapy or radiotherapy, but after surgery, they received chemotherapy (chemotherapy regimens: FOLFOX4 program).

\section{Immunohistochemistry (IHC)}

Tissue sections of $4 \mu \mathrm{m}$ were prepared. IHC was performed by using the Elivision plus kit purchased from MaiXin (Fuzhou, People's Republic of China). The tissue sections were deparaffinized by using xylene and were rehydrated with graded alcohol. Antigen retrieval was performed in citrate buffer ( $\mathrm{pH}$ 6.0) for $2 \mathrm{~min}$ with an autoclave. $\mathrm{H}_{2} \mathrm{O}_{2}$ was used to block the endogenous peroxidase. Normal goat serum was used to reduce nonspecific binding. Then, the sections were incubated with WWC3 rabbit polyclonal antibody (1:200 dilution; Sigma-Aldrich, St Louis, MO, USA) overnight at $4^{\circ} \mathrm{C}$. Rabbit immunoglobulin (at the same concentration as for the antigen-specific antibody) was used as a negative control. The staining was followed by incubation with polymer secondary antibodies. The peroxidase reaction was developed with DAB (3-3'diaminobenzidine tetrachloride) plus from MaiXin. Counterstaining was done with hematoxylin, and the sections were dehydrated in alcohol before mounting.

Two independent investigators examined all tumor slides randomly. Five views were examined per slide, and 100 cells were observed per view at $400 \times$ magnification. Immunostaining of WWC3 was scored following a semiquantitative scale by evaluating the intensity and percentage of tumor cells in representative tumor areas. Cytoplasmic immunopositivity was considered as positive WWC3 staining. The intensity of WWC3 staining was scored as 0 (negative), 1 (weak), 2 (moderate), and 3 (strong). Percentage scores were assigned as 1 for $1 \%-25 \%, 2$ for $26 \%-50 \%, 3$ for $51 \%-75 \%$, and 4 for $76 \%-100 \%$. The scores of each tumor sample were multiplied to give a final score of $0-12$. Tumors were determined as low expression (final score $<6$ ) or high expression (final score $\geq 6$ ).

\section{Cell culture and transfection}

SGC-7901, AGS, HGC-27, and BGC-827 cell lines were obtained from American Type Culture Collection (Manassas, VA, USA). GES-1 cell line was obtained from the Institute of Biochemistry and Cell Biology, Chinese Academy of Sciences (Shanghai, People's Republic of China). The cells were cultured in DMEM (Dulbecco's Modified Eagle Medium; Thermo Fisher Scientific, Waltham, MA, USA) containing 10\% fetal bovine serum (FBS, Thermo Fisher Scientific).

WWC3 plasmid was obtained from OriGene (Rockville, MD, USA). Lipofectamine 3000 transfection reagent was used for plasmid transfection (Thermo Fisher Scientific). The empty vector (pCMV6) was used as negative control.

\section{Small interfering RNA (siRNA) treatment} siGENOME siRNA pool for WWC3 (M-013869-01-0005) and negative control siRNA (D-001206-13-05) were obtained from Dharmacon (GE Healthcare, Lafayette, CO, USA). siRNAs were transfected by using DharmaFECT 1 reagent (GE Healthcare) according to the manufacturer's protocol. Cells were harvested for assessment after $48 \mathrm{~h}$.

\section{Quantitative real-time polymerase chain reaction (PCR; SYBR Green method)}

Real-time PCR was performed by using SYBR Green master mix (TaKaRa, Dalian, People's Republic of China). PCR was 
performed by using 7500 Real-Time PCR System (Thermo Fisher Scientific). $\beta$-Actin was used as the reference gene. The relative expression of target genes was calculated by using the $2^{-\Delta \Delta C t}$ method. Experiments were repeated in triplicate. The primer sequences are listed in Table 1.

\section{Western blot (WB) analysis}

Total proteins from cells were extracted in radio immunoprecipitation assay lysis buffer and quantified by using the Bradford method. Separation of nuclear protein was performed by using NE-PER Nuclear and Cytoplasmic Extraction Reagents (Pierce, Waltham, MA, USA). Protein samples were transferred to polyvinylidene fluoride membranes (EMD Millipore, Billerica, MA, USA) and incubated overnight at $4^{\circ} \mathrm{C}$ with primary antibody against WWC3 (1:500; SigmaAldrich), WWC1 (1:1,000; Sigma-Aldrich), WWC2 (1:800; Sigma-Aldrich), cyclin D1, cyclin E, p27, p21, p53, CTGF, YAP (1:800; Cell Signaling Technology, Boston, MA, USA), and GAPDH (1:2,000; Cell Signaling Technology). After incubation with peroxidase-coupled secondary antibody (1:2,000; Santa Cruz Biotechnology Inc., Dallas, TX, USA) at $37^{\circ} \mathrm{C}$ for $2 \mathrm{~h}$, target proteins on polyvinylidene fluoride membrane were visualized by using ECL kit (Pierce) and DNR Imaging System (DNR, Neve Yamin, Israel).

\section{Colony formation and MTT assays}

For colony formation assay, the cells were transfected with plasmid or siRNA for $48 \mathrm{~h}$ and plated into three 6 - $\mathrm{cm}$ cell culture dishes (1,000 per dish). They were incubated for 12 days in medium containing $10 \%$ FBS. The plates were washed with phosphate-buffered saline (PBS) and stained with Giemsa. The number of colonies with $>50$ cells was counted. The colonies were manually counted by using a microscope.

For MTT assay, $24 \mathrm{~h}$ after plasmid or siRNA transfection, the cells were plated in 96-well plates at a concentration

Table I Primer sequences

\begin{tabular}{ll}
\hline Genes & Sequence $\mathbf{5}^{\prime}$ to $\mathbf{3}^{\prime}$ \\
\hline WWC3 forward & CACGCCCAACACTTACTGCT \\
WWC3 reverse & CGTTAAACACCAGCGGCACT \\
YAP forward & CGCTCTTCAACGCCGTCA \\
YAP reverse & AGTACTGGCCTGTCGGGAGT \\
Cyclin DI forward & TGGAGGTCTGCGAGGAACA \\
Cyclin DI reverse & TTCATCTTAGAGGCCACGAACAT \\
Cyclin E forward & AGCCAGCCTTGGGACAATAAT \\
Cyclin E reverse & GAGCCTCTGGATGGTGCAAT \\
P27 forward & CTGCAACCGACGATTCTTCTACT \\
P27 reverse & CTTCTGAGGCCAGGCTTCTT \\
CTGF forward & GTTACCAATGACAACGCCTCCT \\
CTGF reverse & TGCACTTTTTGCCCTTCTTAATGT \\
Actin forward & TCCACGAAACTACCTTCAACTCC \\
Actin reverse & TCTGCATCCTGTCGGCAAT \\
\hline
\end{tabular}

of $\sim 3,000$ cells per well and cultured for 5 days. For the quantitation of cell viability, $20 \mu \mathrm{L}$ of $5 \mathrm{mg} / \mathrm{mL}$ MTT (thiazolyl blue) solution was added to each well and incubated for $4 \mathrm{~h}$ at $37^{\circ} \mathrm{C}$. The medium was removed from each well, and $150 \mu \mathrm{L}$ of dimethyl sulfoxide was added to the well. The plate was detected using a plate reader at the wavelength of $490 \mathrm{~nm}$.

\section{Cell cycle analysis}

The cells were harvested and fixed by using $1 \%$ paraformaldehyde $48 \mathrm{~h}$ after transfection. Then the cells were washed with PBS and stained with $5 \mathrm{mg} / \mathrm{mL}$ propidium iodide for $30 \mathrm{~min}$ at room temperature. Flow cytometry was performed by using BD FACSCalibur flow cytometer systems (Becton Dickinson, Franklin Lakes, NJ, USA). Cell cycle analysis was carried out by using FlowJo software.

\section{Transwell cell invasion assay}

Cell invasion assay was performed by using a Transwell chamber. The inserts were coated with $18 \mu \mathrm{L}$ Matrigel (BD Biosciences, San Jose, CA, USA) with a dilution rate of 1:4. The cells were plated in a 24 -well plate at $80 \%$ confluence, and siRNA/plasmid transfection was performed. The cells were trypsinized $48 \mathrm{~h}$ after the siRNA transfection, and $100 \mu \mathrm{L}$ of serum-free DMEM was used to dilute the cells. The cells were then suspended and transferred to the upper chamber in $100 \mu \mathrm{L}$ serum-free medium and incubated for $20 \mathrm{~h}$. DMEM with $15 \%$ FBS was added to the lower chamber. The cells passed through the filter were fixed, stained with hematoxylin, and counted under microscope.

\section{Luciferase reporter assay}

To assess the functional regulation of YAP as a transcription coactivator, luciferase assay was performed according to the manufacturer's protocol (Promega Corporation, Fitchburg, WI, USA). The cells were transfected with a combination of plasmids including luciferase reporter plasmid 8xGTIICluciferase, WWC3 plasmid, and WWC3 siRNA. After $48 \mathrm{~h}$, the cells were analyzed for luciferase activities.

\section{Statistical analysis}

SPSS version 16 (SPSS Inc., Chicago, IL, USA) was used for statistical analysis. $\chi^{2}$ test was used to examine possible correlations between WWC3 expression and clinicopathological factors. The Kaplan-Meier method was used to estimate the probability of patient survival, and differences in the survival of subgroups of patients were compared by using Mantel's log-rank test. The Cox regression model was used for multivariate analysis. Differences between transfection/control groups were compared by using Student's $t$-test, and $P<0.05$ was considered statistically significant. 


\section{Results}

\section{Clinical significance of WWC3 in GC tissues}

IHC was carried out to examine WWC3 expression in 111 cases of GC and 15 cases of normal tissues (Figure 1A-E). First, the specificity of the antibody was validated using GC tissue, corresponding to normal tissue, and cell lines with WWC 3 overexpression and siRNA depletion. WB was used to examine WWC3 with this antibody, which was used in both IHC and WB experiments. As shown in Figure S1A, the WB bands of the cells were strong when WWC 3 was overexpressed and were weak when depleted. GC tissues and normal tissues showed similar single bands at the same molecular weight. These results suggested that the antibody used in IHC and WB was specific for WWC3. WWC3 staining was mainly located in the cytoplasm. Differentiated surface epithelia and mucosal glands in normal gastric mucosa showed strong cytoplasmic staining while 48 of 111 primary GCs showed downregulated WWC3 expression. A section containing both cancer tissue and its corresponding normal adjacent tissue was also used. Differential expression of WWC3 could be observed (Figure 1E). The correlation of WWC3 status with clinicopathological characteristics is shown in Table 2. WWC3 downregulation significantly correlated with advanced TNM (tumor, node, metastases) stage ( $P=0.0005$ ). The rate of WWC3 downregulation in T3-T4 group (49.3\%)
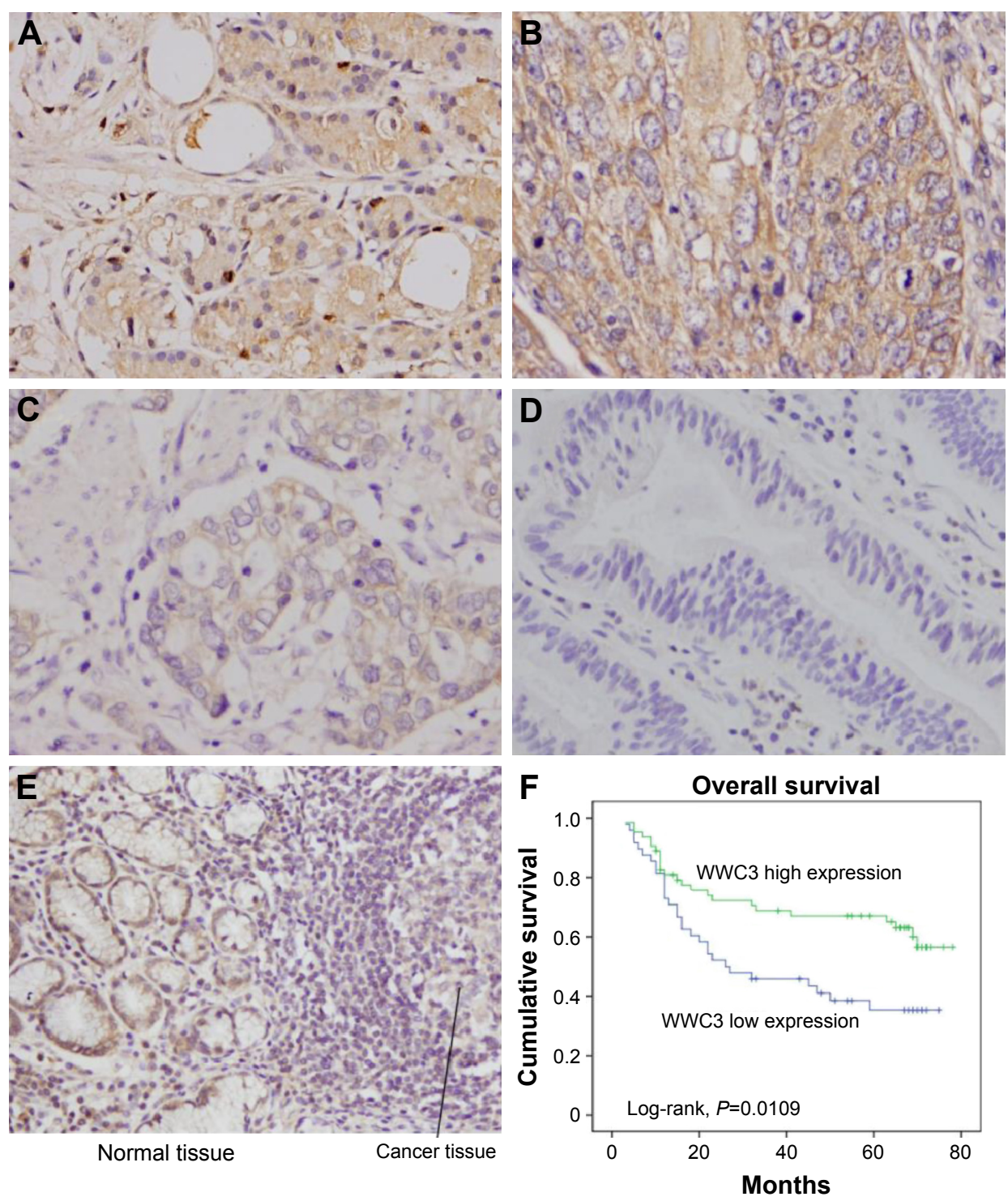

Figure I Expression pattern of WWC3 protein in gastric cancer tissues.

Notes: (A) Positive cytoplasmic WWC3 staining in normal gastric tissues. (B) Positive WWC3 overexpression in a case of adenocarcinoma. (C) Weak WWC3 expression in a case of tubular adenocarcinoma. (D) Negative WWC3 expression in gastric cancer (magnification, 400x). (E) One section containing both cancer tissue and its corresponding normal adjacent tissue was used. Lower expression of WWC3 could be observed in cancer tissue (magnification, I00×). (F) Kaplan-Meier analysis showed that patients with low WWC3 had worse overall survival than patients with high WWC3 protein. 
Table 2 Distribution of WWC3 status in gastric cancer according to clinicopathological characteristics

\begin{tabular}{|c|c|c|c|c|}
\hline Characteristics & $\begin{array}{l}\text { Number } \\
\text { of patients }\end{array}$ & $\begin{array}{l}\text { WWC3 low } \\
\text { expression }\end{array}$ & $\begin{array}{l}\text { WWC3 high } \\
\text { expression }\end{array}$ & $P$-value \\
\hline \multicolumn{4}{|l|}{ Age (years) } & 0.8437 \\
\hline$<60$ & 52 & 23 & 29 & \\
\hline$\geq 60$ & 59 & 25 & 34 & \\
\hline \multicolumn{4}{|l|}{ Gender } & 0.8848 \\
\hline Male & 84 & 36 & 48 & \\
\hline Female & 27 & 12 & 15 & \\
\hline \multicolumn{4}{|l|}{ Differentiation } & 0.6412 \\
\hline Poor & 55 & 25 & 30 & \\
\hline Well-moderate & 56 & 23 & 33 & \\
\hline \multicolumn{4}{|c|}{ Tumor invasion (T) } & 0.0864 \\
\hline $\mathrm{TI}+\mathrm{T} 2$ & 40 & 13 & 27 & \\
\hline $\mathrm{T} 3+\mathrm{T} 4$ & 71 & 35 & 36 & \\
\hline \multicolumn{4}{|c|}{ Lymph node metastasis } & 0.0076 \\
\hline Absent & $4 I$ & 11 & 30 & \\
\hline Present & 70 & 37 & 33 & \\
\hline \multicolumn{4}{|l|}{ TNM stage } & 0.0005 \\
\hline I+II & 51 & 13 & 38 & \\
\hline III & 60 & 35 & 25 & \\
\hline \multicolumn{4}{|l|}{ Relapse } & 0.0109 \\
\hline Negative & 40 & 10 & 30 & \\
\hline Positive & 55 & 28 & 27 & \\
\hline
\end{tabular}

Abbreviation: TNM, tumor, node, metastases.

was higher than that in $\mathrm{T} 1-\mathrm{T} 2$ group $(32.5 \%)$, which did not reach a statistical significance $(P=0.0864)$. The rate of WWC3 downregulation was higher in cancers with positive nodal metastasis $(P=0.0096)$ than those without. In addition, WWC3 downregulation correlated with higher relapse rate ( $P=0.0199)$, and no significant correlation was found between WWC3 downregulation with age and gender.

The Kaplan-Meier analysis showed that patients with low WWC3 expression had shorter overall survival than those with high WWC3 expression (Figure 1F) (overall survival: $P=0.0109)$. Univariate analysis revealed that WWC3 ( $P=0.0133)$, relapse $(P<0.001)$, T stage $(P=0.0026)$, and nodal status $(P<0.001)$ were statistically correlated with patients' survival. These parameters were further subjected to a multivariate Cox proportional hazards model, which indicated that $\mathrm{T}$ stage $(P=0.0359)$, nodal status $(P=0.0137)$, and relapse $(P<0.001)$ were independent and significant factors for prognosis (Table 3 ). Taken together, these data suggest that WWC3 downregulation is an indicator of malignant phenotype and poor prognosis.

\section{WWC3 downregulation accelerates cell proliferation and invasion}

Next, the protein and mRNA levels of WWC3 were analyzed in a panel of GC cell lines (SGC-7901, AGS, HGC-27, and BGC-827) and normal cell line GES-1. It was found that the expression of WWC3 was higher in normal GES-1 cell line than that in GC cell lines. In these cell lines, HGC-27 cells showed relatively low WWC3 level and SGC-7901 cells showed relatively high endogenous level (Figure 2A). WWC1 and WWC2 protein expressions in these similar cell lines were also examined. As shown in Figure S1B, WWC1 was downregulated in AGS and SGC-7901 cell lines compared with GES-1 cell line and WWC2 was downregulated in HGC-27 cell line. WWC3 plasmid was transfected in HGC-27 cell line and siRNA in SGC-7901 cell line. As shown in Figure 2B, WWC3 transfection significantly upregulated its protein and mRNA levels (WWC3 mRNA: empty vector vs plasmid, $P<0.001)$, and siRNA treatment showed the opposite effects (WWC3 mRNA: control vs WWC3 siRNA, $P<0.001)$. The knockdown and overexpression efficiency were also checked at different time points (at 2, 4, and 8 days after transfection) by using WB. As shown in Figure S1C, the transfection of plasmid significantly upregulated WWC3 protein at day 8 . The knockdown efficiency was most significant at day 2 and could still be observed at day 8 .

MTT and colony formation assays were performed to explore the impact of WWC3 on cell proliferation. The results showed that WWC3 overexpression in HGC-27 cells inhibited proliferation rate while siRNA treatment caused an increase in proliferation rate in SGC-7901 cells

Table 3 Univariate and multivariate analyses for predictive factors in patients with gastric carcinoma (Cox regression model)

\begin{tabular}{|c|c|c|c|c|}
\hline \multirow[t]{2}{*}{ Factors } & \multicolumn{2}{|l|}{ Univariate } & \multicolumn{2}{|l|}{ Multivariate } \\
\hline & $\begin{array}{l}\text { Hazard ratio } \\
(95 \% \mathrm{CI})\end{array}$ & $P$-value & $\begin{array}{l}\text { Hazard ratio } \\
(95 \% \mathrm{CI})\end{array}$ & $P$-value \\
\hline Age & $1.250(0.729-2.144)$ & 0.4177 & $1.181(0.676-2.064)$ & 0.5589 \\
\hline Gender & $0.911(0.488-1.700)$ & 0.7685 & $0.789(0.410-1.516)$ & 0.4761 \\
\hline T stage & $1.606(1.180-2.185)$ & 0.0026 & I.43I (I.024-I.999) & 0.0359 \\
\hline Nodal status & I.63I (1.297-2.052) & $<0.001$ & $1.410(1.073-1.854)$ & 0.0137 \\
\hline WWC3 & $0.7 I I(0.543-0.931)$ & 0.0133 & I.I 07 (0.82I-I.494) & 0.5042 \\
\hline Differentiation & $0.750(0.439-1.279)$ & 0.2906 & $0.679(0.383-1.205)$ & 0.1861 \\
\hline Recurrence & $25.01(9.413-66.45)$ & $<0.001$ & $28.913(9.677-86.384)$ & $<0.001$ \\
\hline
\end{tabular}

Abbreviation: $\mathrm{Cl}$, confidence interval. 
(Figure 2C). In accordance, colony formation assay demonstrated that WWC3 overexpression inhibited colony number in HGC-27 cells while its depletion upregulated the colony formation ability in SGC-7901 cells (Figure 2D). Matrigel invasion assay was carried out to assess the role of WWC3 on cell invasion. As shown in Figure 3A, WWC3 overexpression inhibited while its depletion facilitated GC cell invasion.

\section{WWC3 inhibits cell cycle progression and cell cycle-related proteins}

The cell cycle analysis was also performed to observe the impact of WWC3 on cell cycle progression (Figure 3B).
On the one hand, WWC3 overexpression downregulated G1 phase percentage while upregulated $\mathrm{S}$ phase percentage in HGC-27 cells. On the other hand, WWC3 siRNA upregulated G1 phase percentage while downregulated S phase percentage in SGC-7901 cells. These results indicate that WWC3 participates in the cell cycle control of GC. Then, the change of cell cycle-related proteins was checked. As shown in Figure 4A and B, WWC3 overexpression downregulated protein and mRNA expression of cyclin D1 and cyclin E while upregulated p27 in HGC-27 cell line. WWC3 depletion exhibited the opposite effects on these proteins in SGC-7901 cell line. The changes of p21 and p53 protein expression were not significant.
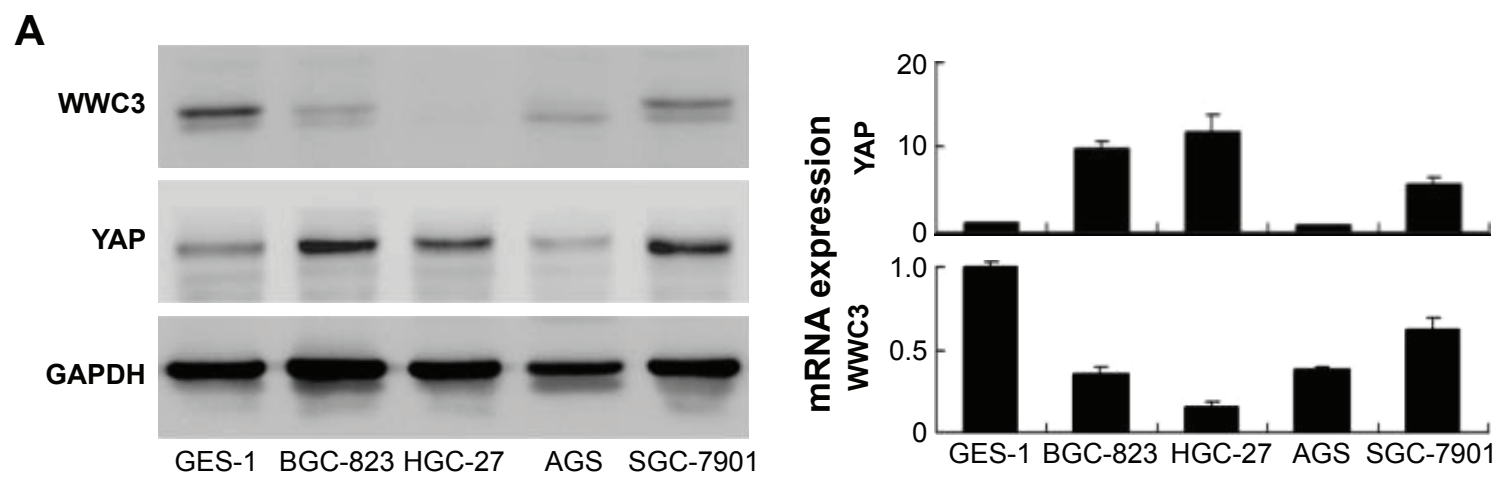

B
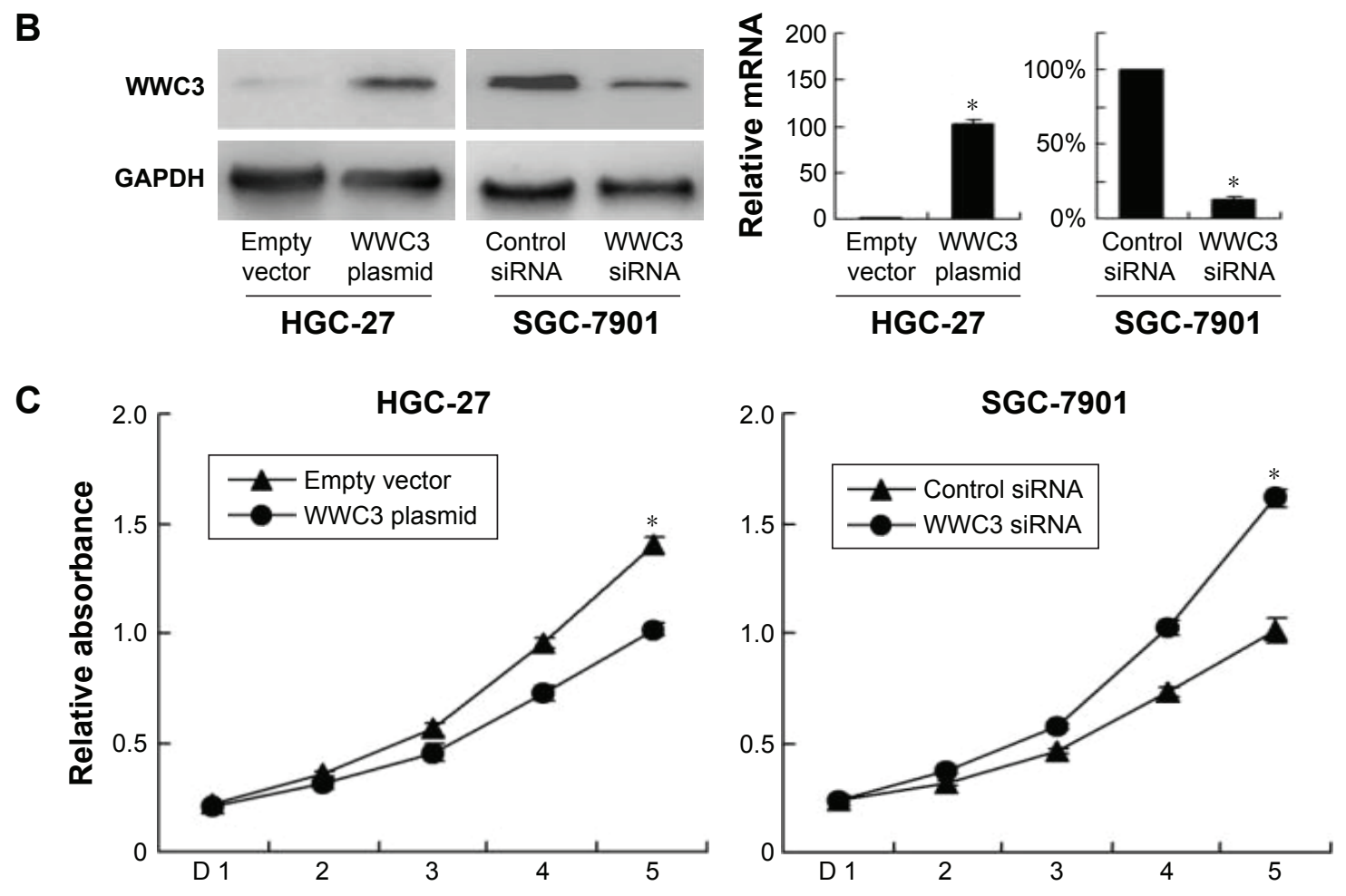

Figure 2 (Continued) 


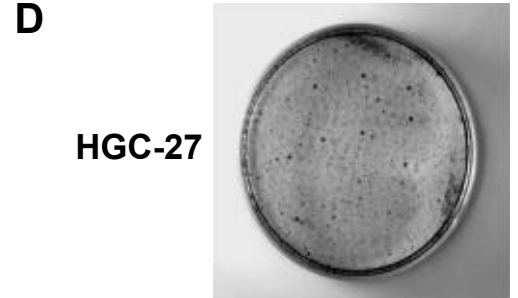

Empty vector

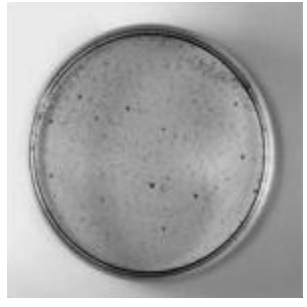

WWC3 plasmid
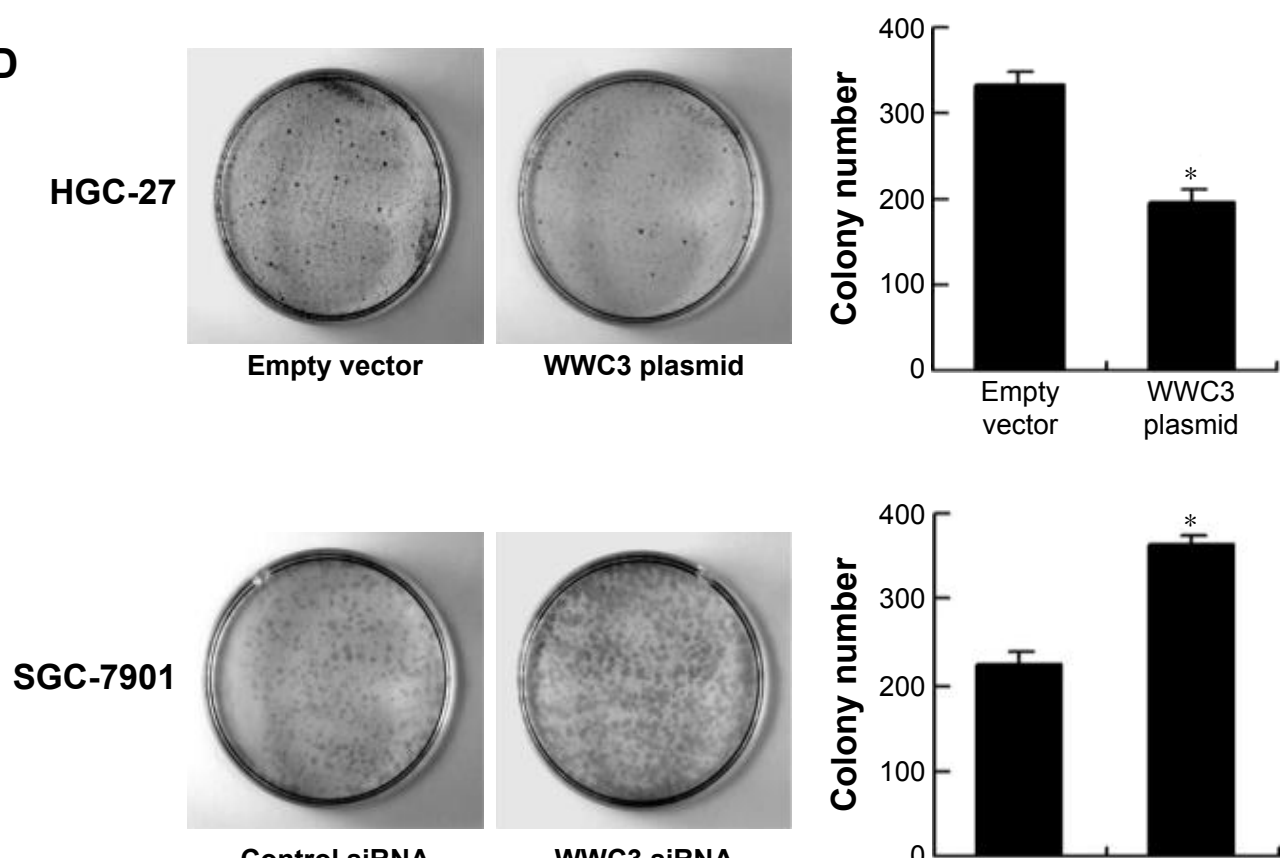

Control siRNA

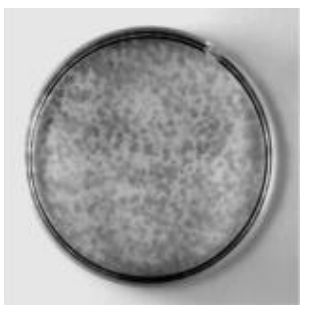

WWC3 siRNA

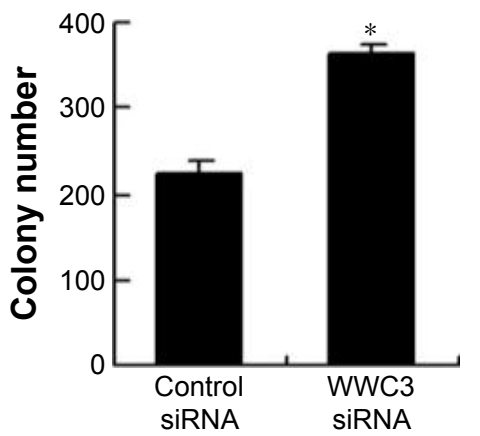

Figure 2 WWC3 inhibits gastric cancer cell proliferation.

Notes: (A) Protein and mRNA expression of WWC3 and YAP in gastric cancer cell lines was lower than that in normal GES-I cell line. HGC-27 cell line has relatively low expression and SGC-790 I cell line has relatively high expression. (B) Western blot and PCR analysis showed that plasmid transfection significantly increased WWC3 level in HGC-27 cells and siRNA decreased WWC3 expression in SGC-790I cells. (C) MTT assay showed that WWC3 overexpression inhibited the proliferation rate in HGC-27 cell line. WWC3 knockdown promoted proliferation rate in SGC-790I cell line. (D) Colony formation assay showed that WWC3 knockdown decreased colony numbers while its overexpression increased colony formation ability. $* P<0.05$.

Abbreviations: siRNA, small interfering RNA; PCR, polymerase chain reaction.

\section{WWC3 overexpression regulates Hippo signaling pathway}

A previous study suggested that WWC family proteins were involved in Hippo signaling pathway. ${ }^{11}$ Hence, the change of YAP, an important Hippo regulator, and its target protein CTGF in WWC3-overexpressed and -depleted cells was examined. First, YAP mRNA and protein levels in all cell lines found in Figure 2A were determined. The results showed that YAP expression in GC cell lines (BGC-823, HGC-27, and SGC-7901) was higher than that in normal GES-1 cell line. Next, it was found that YAP and CTGF protein levels in HGC-27 cells were decreased when transfected with WWC3 plasmid, whereas their levels increased in WWC3-depleted SGC-7901 cell line (Figure 4A and B). Since YAP functions as a transcription coactivator in the nucleus, nuclear YAP was also checked and it was found that WWC3 overexpression inhibited its nuclear localization (Figure 4A). Inhibition of Hippo signaling leads to YAP upregulation, which induces binding of TEAD to target gene promoter. Thus, luciferase reporter assay was used to indicate transcriptional activity of YAP/TEAD. The results showed that WWC3 overexpression downregulated while its depletion upregulated luciferase activity in GC cells (Figure 4C).

\section{Discussion}

GC has posed a great threat to human health all over the world. Although research studies have found that many aberrantly expressed genes in GC can contribute to the malignant behavior, the identification of novel molecular markers is still urgently needed. ${ }^{17,18}$

The WWC family protein has been implicated in human cancers. It was shown that WWC1/KIBRA regulates Hippo signaling in breast cancer and correlates with claudin-low subtype, an aggressive subgroup with EMT features and a poor prognosis. ${ }^{12} \mathrm{WWC1}$ methylation, which is responsible for its downregulation, occurs frequently in B-cell acute lymphocytic leukemia but not in epithelial cancers. ${ }^{15}$ There are no reports regarding protein expression pattern and biological roles of WWC3 in human cancers including GC. For the first time, it has been demonstrated that WWC3 was downregulated in GC tissues and cell lines, which positively correlated with advanced clinical stage, positive nodal status, and higher relapse rate. 
A

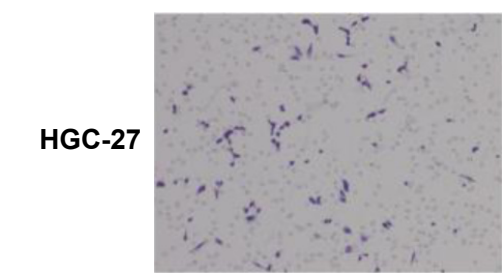

Empty vector

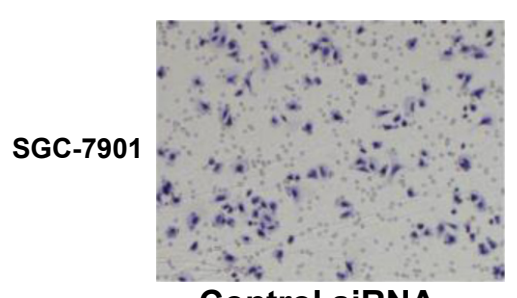

Control siRNA

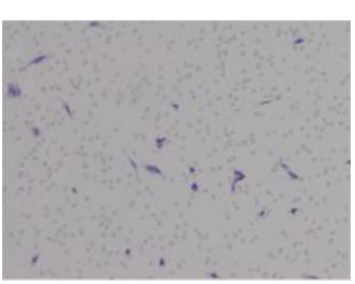

WWC3 plasmid

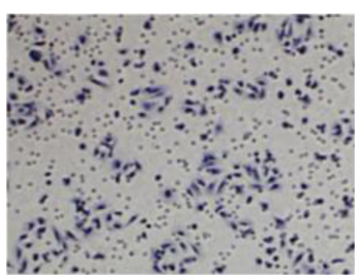

WWC3 SIRNA
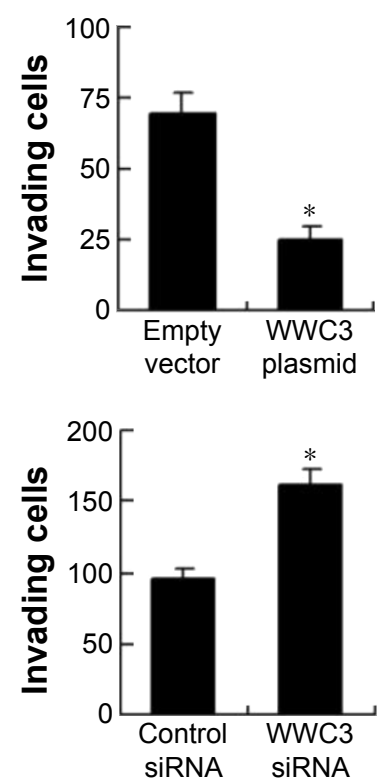

B
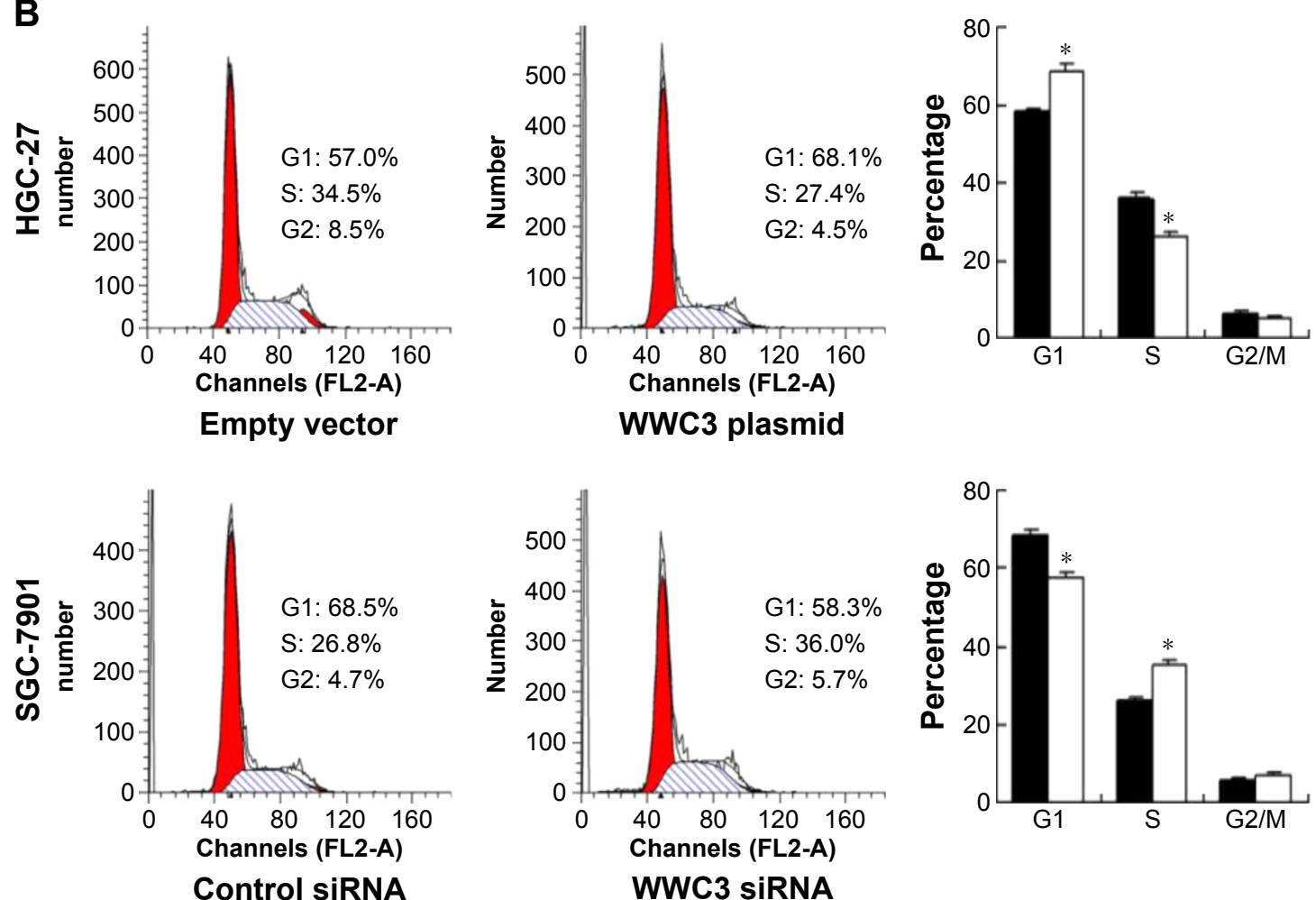

Figure 3 WWC3 regulates gastric cancer cell invasion and cell cycle progression.

Notes: (A) Matrigel invasion assays showed that WWC3 overexpression inhibited HGC-27 cell invasion while WWC3 knockdown promoted SGC-790 cell invasion. (B) WWC3 overexpression increased the percentage of GI cells and decreased the percentage of S phase cells. Conversely, WWC3 siRNA treatment decreased GI phase cells and increased $S$ phase cells compared with control. $* P<0.05$.

Abbreviation: siRNA, small interfering RNA.

Importantly, GC patients with low WWC3 expression showed significantly decreased survival than those with high WWC3 expression. To date, this is the first report regarding the clinical significance of WWC3 in human cancers. The clinical data also demonstrate that loss of WWC3 might serve as a signature predicting and providing prognostic information.
MTT and colony formation assays revealed that WWC3 overexpression led to growth inhibition while its depletion facilitated cell growth. Cell cycle analysis demonstrated that WWC3 overexpression decreased S phase percentage and increased G1 phase percentage. WB showed that WWC3 inhibited cyclin D1 and cyclin E while upregulated p27. 
A

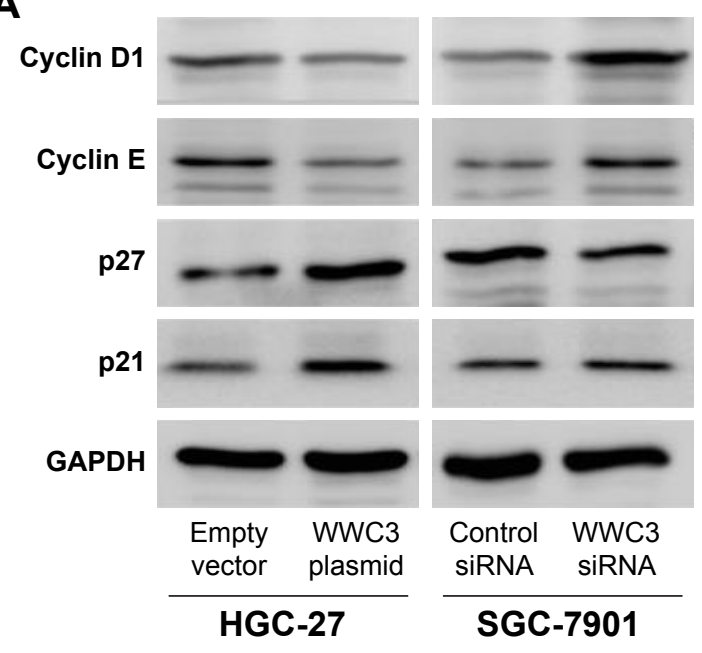

B

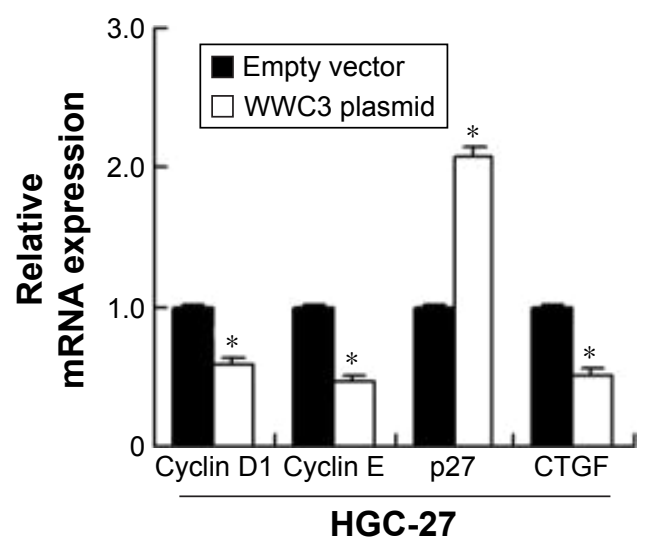

C

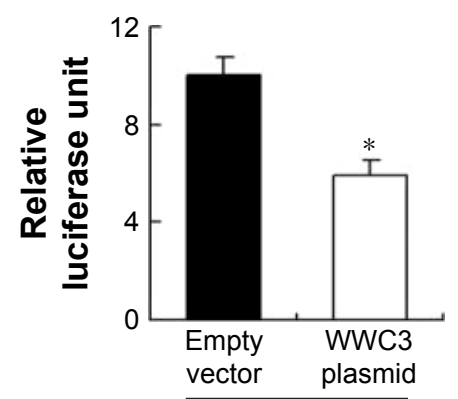

HGC-27
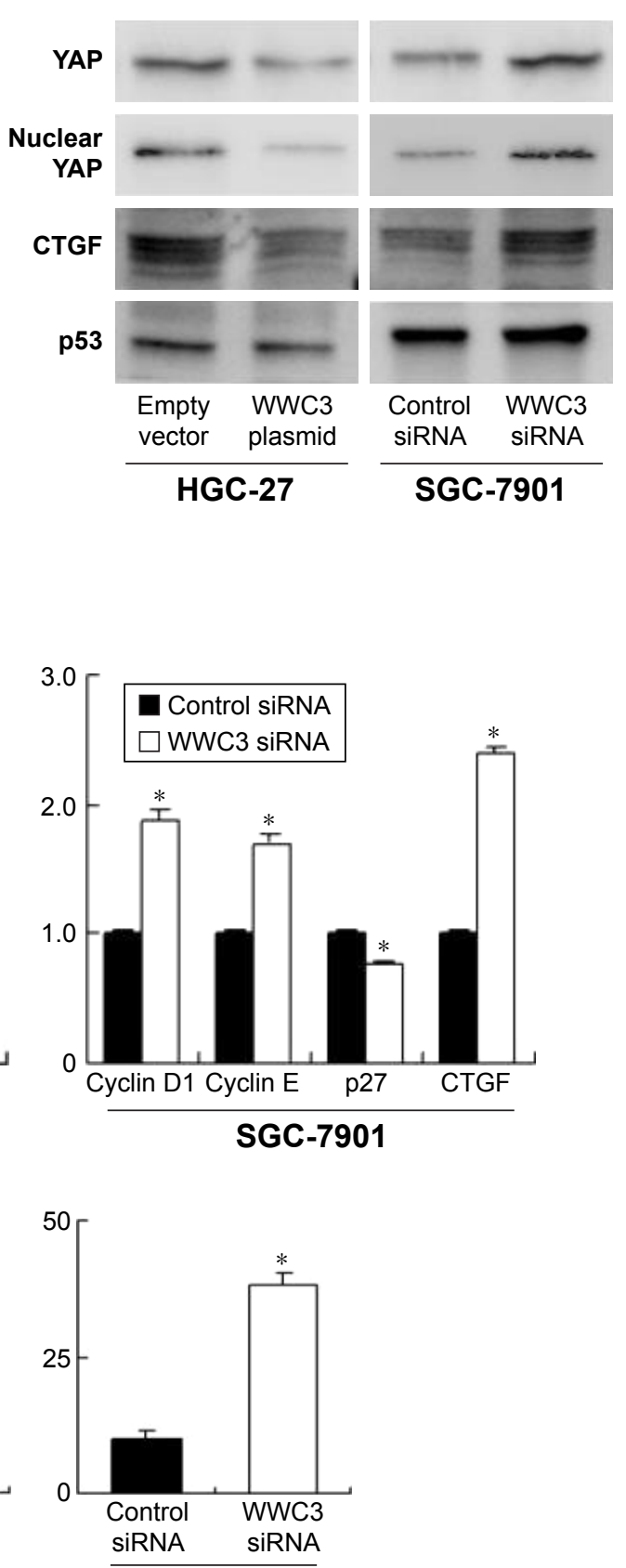

SGC-7901

Figure 4 WWC3 regulates cell cycle-related proteins and Hippo signaling pathway.

Notes: (A) WWC3 overexpression downregulated cyclin DI, cyclin E, CTGF, total YAP, and nuclear YAP and upregulated p27 level in HGC-27 cells. Knockdown of WWC3 upregulated the protein levels of cyclin DI, cyclin E, CTGF, total YAP, and nuclear YAP and downregulated p27 expression in SGC-790I cells. The change of p2I and $\mathrm{p} 53$ was not significant. (B) Real-time PCR analysis showed that WWC3 overexpression downregulated cyclin DI, cyclin E, and CTGF and upregulated p27 mRNA in HGC-27 cells. Knockdown of WWC3 upregulated the protein levels of cyclin DI, cyclin E, and CTGF mRNA and downregulated p27 mRNA in SGC-790I cells. (C) Luciferase reporter assay showed that WWC3 overexpression downregulated while its depletion upregulated 8xGTIIC luciferase activity, which indicates the YAP/TEAD transcription activity. ${ }^{*} P<0.05$.

Abbreviation: PCR, polymerase chain reaction.

Cyclin D1 and cyclin E promote G1-S transition and serve as indicators for malignant growth in various cancers including GC. ${ }^{19,20}$ These results indicate that WWC3 inhibits cell growth by blocking G1-S cell cycle transition. Matrigel invasion assay showed that WWC3 inhibited cell invasion while its depletion promoted cell invasion. Thus, WWC3 could inhibit both proliferation and invasion of GC cells, which was in accord with the clinical data.

The Hippo pathway controls tissue growth and tumorigenesis by inhibiting cell proliferation and promoting apoptosis. To explore the molecular mechanism of WWC3 in GC, Hippo-related proteins were checked and it was 
found that WWC3 overexpression downregulated total YAP, nuclear YAP, and target protein CTGF. WWC3 depletion upregulated YAP and CTGF. YAP and its target protein CTGF are important effector proteins in the Hippo pathway, which have been found dysregulated in various human cancers. ${ }^{21-24}$ The observation of this study was in agreement with previous reports showing correlation between WWC protein and Hippo signaling. YAP overexpression leads to the upregulation of cyclin D1 and concomitant downregulation of $\mathrm{p} 27 / \mathrm{p} 21 .{ }^{25} \mathrm{CTGF}$ is a YAP target protein which plays a critical role in cancer cell proliferation and invasion. ${ }^{26-29}$ Collectively, these results indicate that WWC3 inhibits cell proliferation by downregulating YAP and its target protein CTGF, which in turn inhibits cell cycle proteins and cancer proliferation.

To date, most of the studies regarding WWC1/KIBRA and human cancer considered WWC1 as a tumor suppressor through the activation of Hippo signaling. ${ }^{11}$ However, a recent study suggested a different role of WWC1/KIBRA as a tumor promoter in prostate cancer. WWC1/KIBRA is induced by androgen signaling and is partially required for androgen receptor signaling activation in prostate cancer cells. ${ }^{30}$ Thus, the tumor promoting role of WWC1 is dependent on androgen receptor signaling. WWC1 has a sequence identity of $40 \%$ with WWC3. WWC1 contains a specific, glutamic acid-rich adjacent region, whereas WWC3 contains an arginine- and proline-rich stretch sequence stretch. ${ }^{11}$ Since WWC3 shares similar structure with WWC1, it is possible that the function of WWC3 in other cancers (such as prostate cancer) may differ from that in GC, which deserves further investigation.

\section{Conclusion}

WWC3 downregulation correlates with malignant phenotype and poor prognosis in human GC. WWC3 activates Hippo signaling pathway by the downregulation of YAP protein.

\section{Disclosure}

The authors report no conflicts of interest in this work.

\section{References}

1. Bray F, Ren JS, Masuyer E, Ferlay J. Global estimates of cancer prevalence for 27 sites in the adult population in 2008. Int J Cancer. 2013;132(5): 1133-1145.

2. Jia B, Liu H, Kong Q, Li B. RKIP expression associated with gastric cancer cell invasion and metastasis. Tumour Biol. 2012;33(4):919-925.

3. Jia Y, Dong B, Tang L, et al. Apoptosis index correlates with chemotherapy efficacy and predicts the survival of patients with gastric cancer. Tumour Biol. 2012;33(4):1151-1158.

4. Oishi Y, Watanabe Y, Yoshida Y, et al. Hypermethylation of Sox 17 gene is useful as a molecular diagnostic application in early gastric cancer. Tumour Biol. 2012;33(2):383-393.
5. Janjigian YY, Werner D, Pauligk C, et al. Prognosis of metastatic gastric and gastroesophageal junction cancer by HER2 status: a European and USA International collaborative analysis. Ann Oncol. 2012; 23(10):2656-2662.

6. Yu J, Cheng YY, Tao Q, et al. Methylation of protocadherin 10, a novel tumor suppressor, is associated with poor prognosis in patients with gastric cancer. Gastroenterology. 2009;136(2):640.e1-651.e1.

7. Mayer B, Funke I, Johnson JP. High expression of a Lewis(x)-related epitope in gastric carcinomas indicates metastatic potential and poor prognosis. Gastroenterology. 1996;111(6):1433-1446.

8. Rosse C, Formstecher E, Boeckeler K, et al. An aPKC-exocyst complex controls paxillin phosphorylation and migration through localised JNK1 activation. PLoS Biol. 2009;7(11):e1000235.

9. Duning K, Schurek EM, Schluter M, et al. KIBRA modulates directional migration of podocytes. J Am Soc Nephrol. 2008;19(10): 1891-1903.

10. Makuch L, Volk L, Anggono V, et al. Regulation of AMPA receptor function by the human memory-associated gene KIBRA. Neuron. 2011; 71(6):1022-1029.

11. Wennmann DO, Schmitz J, Wehr MC, et al. Evolutionary and molecular facts link the WWC protein family to Hippo signaling. Mol Biol Evol. 2014;31(7):1710-1723.

12. Moleirinho S, Chang N, Sims AH, et al. KIBRA exhibits MSTindependent functional regulation of the Hippo signaling pathway in mammals. Oncogene. 2013;32(14):1821-1830.

13. Xiao L, Chen Y, Ji M, Dong J. KIBRA regulates Hippo signaling activity via interactions with large tumor suppressor kinases. $J$ Biol Chem. 2011;286(10):7788-7796.

14. Yu J, Zheng Y, Dong J, Klusza S, Deng WM, Pan D. Kibra functions as a tumor suppressor protein that regulates Hippo signaling in conjunction with Merlin and expanded. Dev Cell. 2010;18(2):288-299.

15. Hill VK, Dunwell TL, Catchpoole D, et al. Frequent epigenetic inactivation of KIBRA, an upstream member of the Salvador/Warts/Hippo (SWH) tumor suppressor network, is associated with specific genetic event in B-cell acute lymphocytic leukemia. Epigenetics. 2011;6(3): 326-332.

16. Shinawi T, Hill V, Dagklis A, et al. KIBRA gene methylation is associated with unfavorable biological prognostic parameters in chronic lymphocytic leukemia. Epigenetics. 2012;7(3):211-215.

17. Jeong SH, Ko GH, Cho YH, et al. Pyrophosphatase overexpression is associated with cell migration, invasion, and poor prognosis in gastric cancer. Tumour Biol. 2012;33(6):1889-1898.

18. Yu HF, Zhao G, Ge ZJ, et al. High RIN1 expression is associated with poor prognosis in patients with gastric adenocarcinoma. Tumour Biol. 2012;33(5):1557-1563.

19. Pradeep A, Sharma C, Sathyanarayana P, et al. Gastrin-mediated activation of cyclin D1 transcription involves beta-catenin and CREB pathways in gastric cancer cells. Oncogene. 2004;23(20):3689-3699.

20. Chetty R, Sitti CW. Cyclin E immunoexpression in gastric cancer does not correlate with clinicopathological parameters. Histopathology. 2003;42(1):66-69.

21. Zhang J, Wang G, Chu SJ, et al. Loss of large tumor suppressor 1 promotes growth and metastasis of gastric cancer cells through upregulation of the YAP signaling. Oncotarget. 2016;7(13):16180-16193.

22. Li N, Yu N, Wang J, et al. miR-222/VGLL4/YAP-TEAD1 regulatory loop promotes proliferation and invasion of gastric cancer cells. Am J Cancer Res. 2015;5(3):1158-1168.

23. Jiao $\mathrm{S}$, Wang $\mathrm{H}$, Shi Z, et al. A peptide mimicking VGLL4 function acts as a YAP antagonist therapy against gastric cancer. Cancer Cell. 2014; 25(2):166-180.

24. Zhou Z, Zhu JS, Xu ZP. RNA interference mediated YAP gene silencing inhibits invasion and metastasis of human gastric cancer cell line SGC-7901. Hepatogastroenterology. 2011;58(112):2156-2161.

25. Hsueh YJ, Chen HC, Wu SE, Wang TK, Chen JK, Ma DH. Lysophosphatidic acid induces YAP-promoted proliferation of human corneal endothelial cells via PI3K and ROCK pathways. Mol Ther Methods Clin Dev. 2015;2:15014. 
26. Kothapalli D, Grotendorst GR. CTGF modulates cell cycle progression in cAMP-arrested NRK fibroblasts. J Cell Physiol. 2000;182(1) $119-126$.

27. Wang R, Ding X, Zhou S, et al. Microrna-26b attenuates monocrotalineinduced pulmonary vascular remodeling via targeting connective tissue growth factor (CTGF) and cyclin D1 (CCND1). Oncotarget. 2016;7(45):72746-72757.

28. Braig S, Wallner S, Junglas B, Fuchshofer R, Bosserhoff AK. CTGF is overexpressed in malignant melanoma and promotes cell invasion and migration. Br J Cancer. 2011;105(2):231-238.
29. Tsai HC, Su HL, Huang CY, Fong YC, Hsu CJ, Tang CH. CTGF increases matrix metalloproteinases expression and subsequently promotes tumor metastasis in human osteosarcoma through downregulating miR-519d. Oncotarget. 2014;5(11):3800-3812.

30. Stauffer S, Chen X, Zhang L, Chen Y, Dong J. KIBRA promotes prostate cancer cell proliferation and motility. FEBS J. 2016;283(10): 1800-1811. 


\section{Supplementary material}

A

WWC3

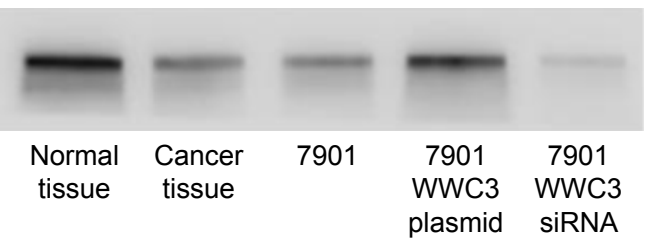

$122 \mathrm{kDa}$

B

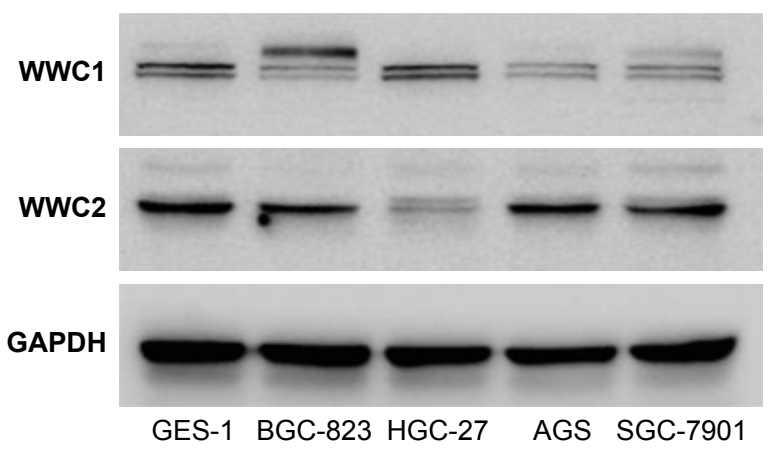

C

Day 2

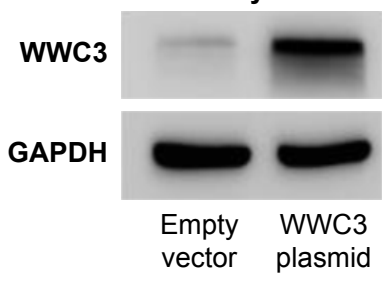

Day 2

WWC3

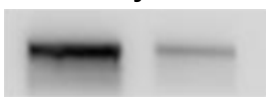

GAPDH

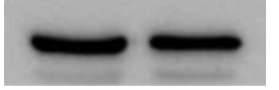

Control WWC3

SiRNA SiRNA
Day 4

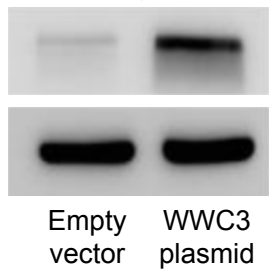

Day 4

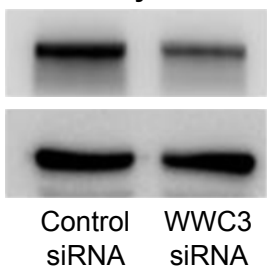

Day 8

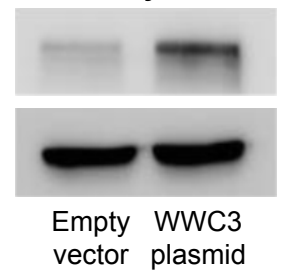

Day 8

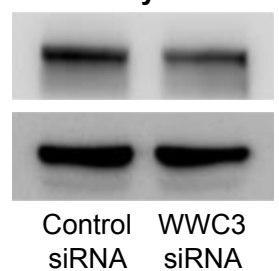

Figure SI Validation of antibody and transfection efficiency.

Notes: $(\mathbf{A})$ The specificity of the antibody was validated by using gastric cancer tissue, corresponding normal tissue, and cell lines with WWC3 overexpression and siRNA depletion. Western blot was used to examine WWC3 with this antibody. The Western blot bands of cells were strong when WWC3 was overexpressed and were weak when depleted. Gastric cancer tissues and normal tissues showed similar single bands at the same molecular weight. (B) Expression of WWCI and WWC2 proteins in gastric cancer cell lines and GES-I cell line. (C) The knockdown and overexpression efficiency were checked at different time points (at 2, 4, and 8 days after transfection) using Western blot. The transfection of plasmid significant upregulated WWC3 protein at day 8 . The knockdown efficiency was most significant at day 2 and could still be observed at day 8.

Abbreviation: siRNA, small interfering RNA.

OncoTargets and Therapy

\section{Publish your work in this journal}

OncoTargets and Therapy is an international, peer-reviewed, open access journal focusing on the pathological basis of all cancers, potential targets for therapy and treatment protocols employed to improve the management of cancer patients. The journal also focuses on the impact of management programs and new therapeutic agents and protocols on

\section{Dovepress}

patient perspectives such as quality of life, adherence and satisfaction. The manuscript management system is completely online and includes a very quick and fair peer-review system, which is all easy to use. Visit http://www.dovepress.com/testimonials.php to read real quotes from published authors. 